\title{
Research on the Application of Reverse Engineering and Material Enhancement in the Shell of Nail Nail Machine Based on Layered Sliced Algorithm
}

\section{Liu Wen}

Department of Aerocarft Manufacturing, Sichuan Aerospace Vocational College, Chengdu, 610100, Sichuan, China

email: tashibuergan@163.com

Keywords: 3D Printing, Slicing, STL Model, MATLAB

\begin{abstract}
In order to improve the slicing efficiency of STL model, a new algorithm based on MATLAB for slicing and stratification of STL model is proposed. The shell of enterprise's new product nailer is selected as the carrier, and the mark point, scanning, point cloud data analysis, surface solid 3D modeling reconstruction and 3D surface innovation design are carried out. Firstly, the STL model is preprocessed to select the set of triangular slices that only intersect the tangent plane, and then the topological relationship of the adjacent triangular face edge is used to obtain the cross section profile information of each layer in turn. The experimental results show that the method is simple and effective, which not only optimizes the data structure, but also improves the slicing efficiency, and has good feasibility and accuracy.
\end{abstract}

\section{Introduction}

3D printing, also known as rapid prototyping technology, is a digital model file based technology, which uses powder film or plastic to construct objects by stacking them, which has remarkable advantages in complex structures, with variable shapes, many skeletal features and difficult to process in human body, so it is widely used in modern medicine. In 3D printing, cutting is one of the most important parts. So that the efficiency and precision of plate processing directly affect the accuracy and quality of the model [1]. In this paper, using MATLAB software as the development platform, the 3D print slicing problem is studied, and some functions of matrix and array and MATLAB are used to realize the rapid layered slicing of STL model under MATLAB software platform.

3D Stereo printing technology, as an important development direction of modern advanced manufacturing technology and leader of the third industrial revolution, is rapidly changing people's traditional mode of production, widely used in various lines. efficiency is a problem that all technical researchers are actively exploring [2]. on this basis, this paper studies the hierarchical model slicing algorithm of stl type documents, selects the new type of nail projectile as the carrier for 3d data processing, and carries out cloud computing. through the analysis of the surface model and the innovative design, a new surface model of nail projectile is proposed, and the triangular disk algorithm is applied to supplement the 3d additional pressure sample [3].

\section{Principle of Layered Slicing Algorithm for Triangulation of STL file}

The STL model is a three-dimensional object consisting of several triangular points. therefore, by cutting the model, several tangent planes along the intersecting direction (z-axis) are determined, and the intersection of each tangent plane or triangulation planning point is determined, and then the topological feedback is carried out to get the contour line.

Because of the large amount of data in the STL model, the following method is used to calculate: first, the data in the STL model is sorted, and then the collection of triangular face pieces cut with each plate is selected to reduce the cross times between the triangular face sheet and the plate [4]. through the topological relationship between the edges of the adjacent triangle face sheet, the intersection of the triangle face sheet and the key plane of each layer is quickly determined to 
reduce the calculation of redundant points.

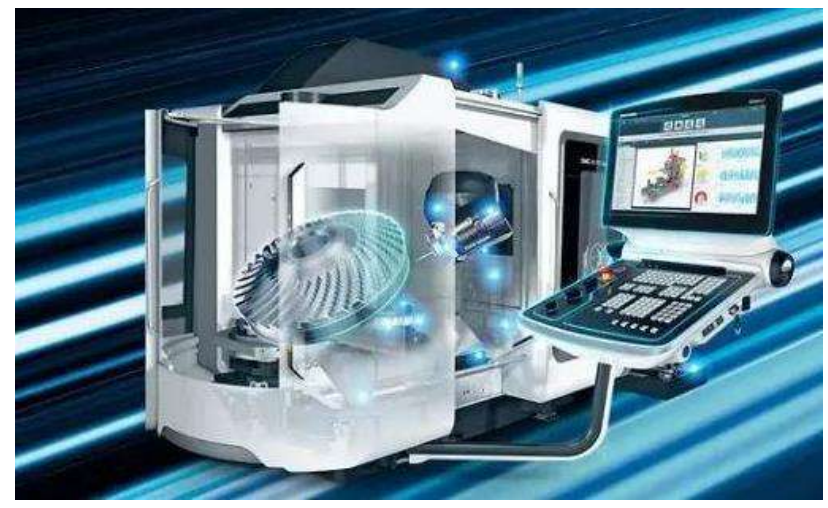

Figure 1 Hierarchical slicing algorithm

\subsection{Data Preprocessing}

Using the stlread function of MATLAB software to read the STL model data, a triangular mesh object is obtained, which contains two matrix members: one is the coordinate matrix of the mesh vertex, set to $\mathrm{P}$, and the other is the vertex index matrix that makes up the triangular face sheet, set to $\mathrm{T}$.

Take the vertex index of each triangular sheet in turn from the triangular sheet index matrix $\mathrm{T}$, and find the $\mathrm{x}, \mathrm{y}$, and $\mathrm{z}$ coordinates of its vertices from the vertex coordinate matrix $\mathrm{P}$, and then sort them according to the size of the $\mathrm{z}$ coordinate values and line up:

[x1, y1, zmin, x2, y2, zmid, x3, y3, zmax]

thus, a new triangular facet coordinate matrix $\mathrm{vm} \times 9$ is obtained, where each row represents a triangular facet coordinate information, and $\mathrm{m}$ is the total triangular facet. the matrix $\mathrm{v}$ is then sorted in ascending order according to zmin, and if zmin is the same, the triangular facet with small zmax values is ranked first.

\subsection{Smart Screening of Intersecting Triangles}

when calculating the intersection of each layer cut plane and the triangular face sheet, judging from the z-coordinate data of each triangular face sheet, the set of triangular face sheet that only intersects the current cut plane is selected to improve the delamination processing speed. The steps are as follows:

step 1, firstly, isothickness delamination is carried out according to the slice accuracy $\Delta \mathrm{z}$ and the maximum and minimum values of the model $z$ coordinates to obtain the height $z i(i=1,2, \ldots, n, n$ for the total number of slices) of each layer cut plane.

in step 2, the judgment condition is used to screen out the triangular facet sheet set intersecting each layer cut plane zi. If the triangle sheet intersects with the cut plane $\mathrm{zi}$, the $\mathrm{zmin} \leq \mathrm{zi} \leq \mathrm{zmax}$ should be satisfied. As shown in Figure 1, it can be divided into the following cases:

two edges of the triangular facet intersect with the slice surface (fig .1(a));(2) one edge of the triangular facet coincides with the tangent plane (fig .1(b)(c)), if fig .1(b) and fig .1(c) have coedges, to avoid generating redundant points, only the triangular sheet shown in fig .1(b) is retained ;(3) a vertex of the triangular facet is on the tangent plane (fig .1(d)(e)), to avoid generating redundant points, only the triangular sheet shown in fig .1(d);(4) the triangle facet and the tangent plane coincide with the tangent plane (fig .1(f), therefore, this situation can not produce effective intersecting lines, and the line segment is excluded. 


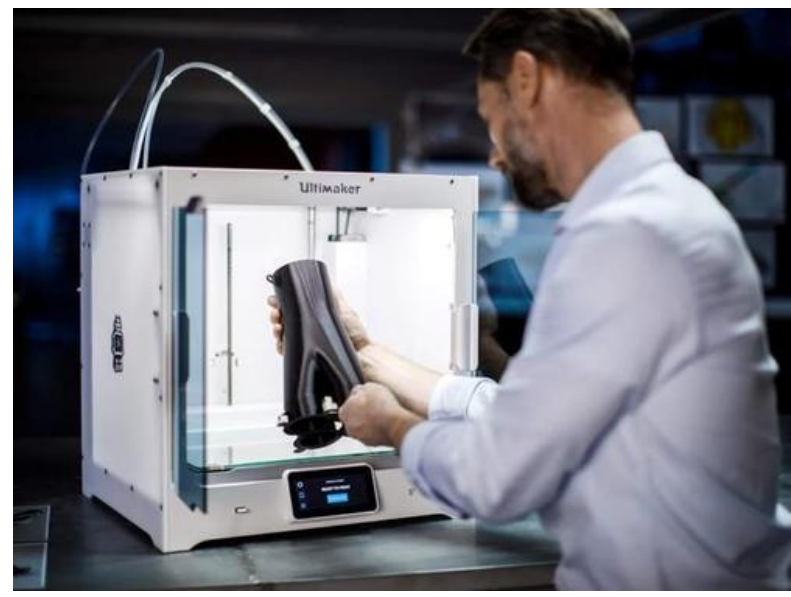

Figure 2 3D Print

\subsection{Topology Intersection}

In the triangular mesh model, each edge has two triangular slices to share with it, so using this adjacency topological relation, we can get the slicing wheel with the end and the end in sequence. The steps are as follows:

step 1, from the selected set of intersecting triangular facets, randomly determine a triangular facet as the starting triangular facet, calculate the intersection of the triangular facet and the tangent plane, and save the edge where the intersection point is located, and then remove this triangular facet from the set.

In step 2, find a triangular face sheet with the intersection point of the previous triangular face sheet as the common edge in the set of intersecting triangular face sheets, and then calculate the intersection point of the triangular face sheet, after saving the edge of the intersection point, remove the triangular face from the set [5]. As shown in Figure 2, according to the edge $A B$ of the intersection point P1 of the triangular facet sheet 1 , find the adjacent triangular facet sheet 2 , then calculate the other intersection point $\mathrm{P} 2$ between the tangent plane and the triangular facet sheet 2, according to the edge $\mathrm{BC}$ where the intersection point $\mathrm{P} 2$ is located, and so on.

step 3, repeat step 2 until the next topological triangular face sheet can not be found, the current closed contour can be obtained by connecting the intersection points in sequence.

step 4, repeat step 1, step 2, step 3 until the intersecting triangular sheet set is an empty set, thus completing the creation of all closed contours of the layer slice.

\section{Instance Validation}

To test the effectiveness of this algorithm, slice the scorpion model shown in Figure 3 . The model has a total of 461708 triangular facets ,230856 vertices, and the overall dimensions in the X, $\mathrm{Y}, \mathrm{Z}$ directions are about $59 \mathrm{~mm}, 42 \mathrm{~mm}, 20 \mathrm{~m}$. slice the model with $0.4 \mathrm{~mm}$ delamination accuracy. from the results of figure 4 , it can be seen that even if the model is complex and has multiple branching structures, the algorithm in this paper can produce better results.

\section{3D Print Common Problem}

In the process of 3D printing, the models we print often have one problem or another. Taking 3D printers made of ABS and PLA as an example, we will encounter the following common phenomena 


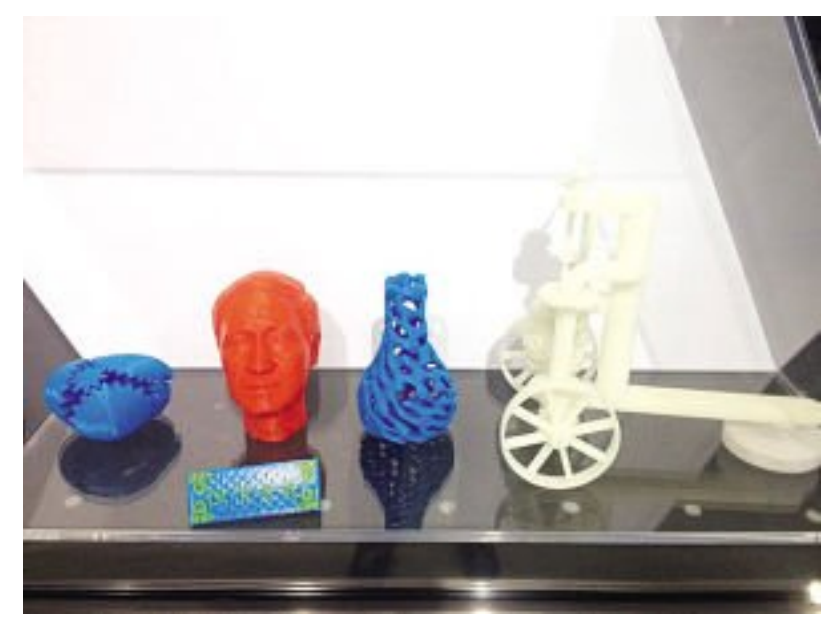

Figure 3 3D Printing

\subsection{Leveling of Platforms}

Before using the 3D printer, first plan the work. The distance between the blowing mouth and the processing platform, so that the blowing mouth keeps a fixed distance with the working platform when moving, if the distance is too small, it is difficult to separate, resulting in the first layer is very thin, affecting the pressure effect; if the distance is too long, the first layer can not paste this is why it is very important to classify the 3D printer. During the leveling process, the lid on the print platform is usually adjusted to change the distance between the blowing mouth and the manual leveling platform [6]. The specific step is to tighten the nut on the platform, place an A4 paper on the platform and run the level program on the printer. When the nozzle moves to a specific position, adjust the nut so that the distance between the platform and the nozzle is exactly as thick as a sheet of paper; gently pull out the paper, adjust it, and then do the next position so that the distance of each position is the same, the platform level, this is a patient's work, must be done multiple times at the first reading, to lay a good foundation for follow-up work [7].

\subsection{Print Model Edging}

In the process of printing, we often come out when printing parts. When the printing time is over, the edge of the model tends to bend. Why? This is because when printing, because of the thermal expansion and cold shrinkage of the material, the material will shrink after coming out, causing frontier war, compared with the small number, the larger the model, the longer the distance, the more clear the stretching and shrinkage, the greater the effect, in the 3D printing process, can be improved from the following aspects to reduce the edge distortion of the wind [8]. In order to reduce the impact and improve the degree of edge fighting, if the equipment does not have the function of hot mode, it can reduce the pressure speed, change the line width of the first layer, and also glue the glue to the platform to increase the coating of the material and avoid the edge deformation.

\subsection{Layering of Print Models}

Because the FDM-3D printer is by melting the material, stacking the material from the inventory to the inventory, forming a variety of complex models, in the process of processing, it is often found that some machine models have a connection when the processing is completed or not. The Huba model is layered at the print arm because there is no good combination between the layers, causing the entire model to open [9]. Check whether the height of the layer is suitable, in general, the height of the layer can not be set larger than the diameter of the blow, the height of the layer will affect the adhesion between the layer and the layer, if the improvement is not enough, can be improved by adjusting the blow temperature; because the blow temperature is too low, the material can not be completely melted, so the material can not be well combined in the cooling process, can adjust 5 degrees at a time to see if the overall treatment is improved, when the model is not high, the layer can be accepted, can be fixed with glue [10]. 


\section{Conclusion}

This paper takes MATLAB software as the platform to cut and cover the model effectively through the steps of preprocessing the data, selecting the triangulation sheet set and selecting the topological intersection point; this method is convenient and quick, improves the controllability of the circuit board, optimizes the working efficiency of the disk, improves the accuracy of the clip board, the program is simple and easy to understand, and has better program value.

The principle of 3D printed STL triangulation average algorithm is studied, and the wheel of the current intersecting view is obtained by connecting the intersection point by using the principle of triangle sheet intersecting with the key plane. the algorithm only needs to read the primary source data from the stl model, and the resulting shear contour layer is very complete and closed, which greatly improves the processing speed of the coating layer during the $3 \mathrm{~d}$ printing process. The high quality closed library CD greatly improves the calculation efficiency of 3D printing, provides new technical support and transformation of production mode for the development of new products by nail pliers, shortens the development cycle of new products, reduces the production cost of new products, and saves the time of opening mold and making mould.

\section{References}

[1] Hu Dezhou, Li Zhanli, Li Dezhen,. Research on fast hierarchical processing algorithm based on geometric feature classification of STL model. Journal of Xi'an Jiaotong University, no. 1, pp. 3740,45, 2000. doi :10.3321/j.issn :0253-987 X.2000.01.009.

[2] Xie Cunxi, Li Zhongyang, Cheng Xiao. Study on the establishment and slicing algorithm of adjacency relation of STL file. Journal of South China University of Technology,no. 3, pp. 33-38, 2000. doi :10.3321/j.issn :1000-565 X.2000.03.007.

[3] Shi Zhenling, Zhou Shiquan, Yu Linbo,. Establishment of STL File Topological Relation and Research on New Triangulation Search Algorithm. New Technologies and New Technologies, no. 7, pp. 14-16, 2006. doi :10.3969/ j.issn.1003-5311.2006.07.004.

[4] Yang Guang, Liu Weijun, Wang Wei,. Topology reconstruction and fast slicing algorithm of STL format files. Modern Manufacturing Engineering, no. 10, pp. 32-35, 2009. doi :10.3969/ j.issn.1671-3133.2009.10.009.

[5] Zhao Jibin, Liu Weijun, Wang Yue. Optimization algorithm study of rapid prototyping slice data. Minicomputer system, no. 10, pp. 1818-1821, 2004. doi :10.3969/ j.issn.1000-1220.2004 10.022 .

[6] Ma Liang, Huang Wei. fast slicing algorithm based on dynamic topology reconstruction of STL data model. China Laser, no. 10, pp. 1623-1626, 2008. doi :10.3321/j.issn :0258-7025.10.037.

[7] Wang Chunxiang, Hao Zhibo, Chen Hao. hierarchical optimization of stl model based on matlab software in rapid prototyping. Machine Tools and Hydraulics, no. 21, pp. 113-117, 2014. doi :10.3969/ j.issn.1001-3881.2014.21.030.

[8] Guo Liang, Anfen Ju, Wang. Portable 3D printer design. Mechanical and Electrical Engineering Technology, vol. 46, no. 10, pp. 90-92+108, 2017.

[9] Xu Jinghua, Ren Xinhua, Chen Qianyong,, manifold model 3d print layer cutting optimization method based on double dip probability. Journal of Mechanical Engineering, vol. 55, no. 13, pp. 129-143, 2019.

[10] Xu Jinghua, Sheng Hongsheng, Zhang Shuyou,. manifold grid model layer cutting multiconnected domain construction method based on adjacency topology. Journal of Computer Aided Design and Graphics, vol. 30, no. 1, pp. 180-190, 2018. 